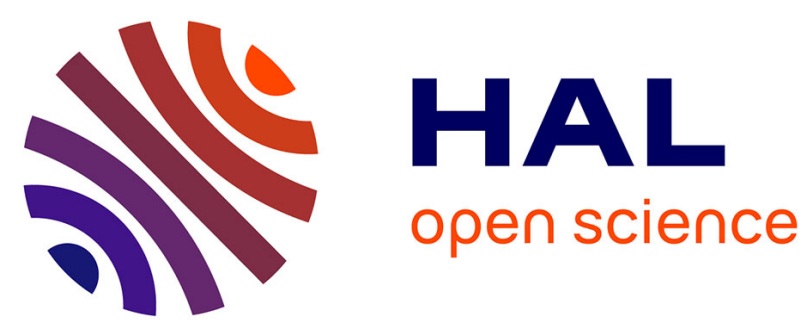

\title{
Importance of the dynamics of adsorption and of a transient interfacial stress on the formation of aggregates of IgG antibodies
}

Sergii Rudiuk, Laetitia Cohen-Tannoudji, Sylvain Huille, Christophe C. Tribet

\section{- To cite this version:}

Sergii Rudiuk, Laetitia Cohen-Tannoudji, Sylvain Huille, Christophe C. Tribet. Importance of the dynamics of adsorption and of a transient interfacial stress on the formation of aggregates of $\operatorname{IgG}$ antibodies. Soft Matter, 2012, 8 (9), pp.2651. 10.1039/c2sm07017k . hal-02369016

\section{HAL Id: hal-02369016 https://hal.science/hal-02369016}

Submitted on 21 Nov 2019

HAL is a multi-disciplinary open access archive for the deposit and dissemination of scientific research documents, whether they are published or not. The documents may come from teaching and research institutions in France or abroad, or from public or private research centers.
L'archive ouverte pluridisciplinaire HAL, est destinée au dépôt et à la diffusion de documents scientifiques de niveau recherche, publiés ou non, émanant des établissements d'enseignement et de recherche français ou étrangers, des laboratoires publics ou privés. 


\title{
Importance of the dynamics of adsorption and of a transient interfacial stress on the formation of aggregates of IgG antibodies
}

\author{
Sergii Rudiuk, ${ }^{a}$ Laetitia Cohen-Tannoudji, ${ }^{b}$ Sylvain Huille ${ }^{b}$ and Christophe Tribet $*^{a}$ \\ Received (in XXX, XXX) Xth XXXXXXXXX 20XX, Accepted Xth XXXXXXXXX 20XX \\ ${ }_{5}$ DOI: 10.1039/b000000x
}

It is common knowledge that aggregation of proteins may occur in aqueous solutions under mechanical stress (shaking or high shear), even in solutions that are stable at rest. Addition of surfactants is a practical generic means to prevent this stress-induced aggregation (e.g. in formulations of therapeutic proteins), which suggests that interfaces contribute to destabilization. We studied here the role of interfacial stress

10 by applying brief mechanical impacts on air-water interface, in presence or absence of surfactants, in solutions of immunoglobulin G (IgG), a class of proteins of high importance to the developments of new therapeutics. A variety of surfactants was tested including the neutral ones Tween80, C10-C14 foscholines, alkylaminoxide, surfactin, and two ionic ones TTAB and lauroylsarcosine sodium salt. We determined the presence of aggregates in solution by light scattering. Irrespective of the type of antibody, 15 either human polyclonal or a monoclonal one, we show that the amount of aggregated IgG increases in proportion to the number of impacts on the interface. In absence of stress, we recorded images of oblate aggregates of IgG (ca. $12 \mathrm{~nm}$ height and 200-1200 nm in diameter) present at the air-water interface (fluorescence microscopy using anti-Fab or anti-Fc markers, and AFM scans after transfer on freshly cleaved mica). Our results evidence that aggregates are formed at air-water interface, and are brought in 20 solution by transient stresses applied on the water surface. Rupture of interfacial films is an important source of aggregates in solution. Finally, the role of surface dynamics in the protection brought by surfactants is discussed based on comparison of protective efficiencies with dynamic surface tension properties (measured by the maximum bubble pressure method). Our work indicates that better protection is conferred by surfactants showing the faster interfacial dynamics, which corresponds also to conditions 25 of faster lowering of the interfacial energy at short time scale.

\section{Introduction}

Application of mechanical stress to protein solutions is often suspected to facilitate the formation of proteins aggregates. Better understanding of the origin of protein aggregation under stress 30 would avoid artefacts or deleterious effects resulting from unexpected aggregation that occur upon shaking, or stirring. For instance, stress-induced aggregation is a question debated as a likely explanation of the slow increase of viscosity with shearing time in solutions of globular protein. ${ }^{1}$ It has been argued that 35 destabilization depends on the nature of interfaces in contact with solutions, and that shear stress is usually not high enough to play a significant role. ${ }^{1}$ Protection against such stresses has also important consequences to preventing hazardous handling of protein solutions, e.g. to avoid fibrillations, ${ }^{2}$ or to safely handle ${ }_{40}$ formulations of biotherapeutics whose aggregates may elicit severe immune responses. ${ }^{3-5}$ Preservation of proteins stability is accordingly a major challenge for the development of recombinant antibodies that are the fastest growing class of these new drugs.

45 At rest monodispersity in aqueous solutions can be preserved even for months, by proper engineering of protein sequences and post-modifications, and optimal choice of storage buffers: the generic routes toward destabilization and aggregation of proteins in bulk are essentially well described. ${ }^{6,7}$ Absence of aggregation 50 in bulk, however does not rule out destabilization and aggregation upon adsorption. Surface aggregation typically evolves during hours on fluid interfaces and can result in clusters of proteins with diameters of several hundreds of nanometers ${ }^{8}$ or fractal structures sticking on the surface. ${ }^{9,10}$ In this context, hydrophobic ${ }_{55}$ binding is generally evoked as the main driving force for non specific aggregation. Namely, conditions destabilizing the native protein, including adsorption, permit transient unfolding and exposure of hydrophobic domains, leading to inter-protein hydrophobic associations. ${ }^{5,10}$

60 Surfactants are well known agents that protect proteins against aggregation. ${ }^{11}$ In the presence of these amphiphilic additives, the association between hydrophobic patches of proteins is expected to be reduced because of the competition with binding of surfactants. ${ }^{12}$ Bound surfactants may for instance form protective 65 layers on hydrophobic patches that become screened from a direct contact with water. This simple mechanism does not 
however suffice to explain the excellent stability achievable with many proteins in solution at rest (absence of degradation for months), while aggregates form rapidly under mechanical stress. $^{12,13}$ It appears thus of importance to investigate on the 5 origin of aggregation occurring upon manipulation of protein solutions, and to find rational origin of the protection brought by amphiphilic stabilizers.

We studied the destabilization of antibodies in aqueous solutions with the aims of i) finding a reproducible test to 10 characterize the aggregation under conditions approaching practical handling and shearing, and ii) analyzing the origin of protection brought by surfactant additives. Here we studied mixtures of surfactants and immunoglobulins G: human polyclonal immunoglobulin (IgGp) or a monoclonal antibody 15 (IgGm) from the LFB Biotechnologies (see experimental section for more details). We characterized by light scattering their stability under mild stirring, with and with no mechanical stress applied on the air-solution interface. In addition, AFM and fluorescence microscopy (FM) were used to characterize the size, 20 shapes and the presence of aggregates on the interface. We compared a set of surfactants with varying chain length and hydrophilic heads, including Tween 80 , a representative additive present in commercial formulations of IgG. Altogether, our results enable us to propose a rational scheme for the transfer of 25 aggregates of IgG in bulk upon mechanical stress on air-water interface, and to raise the question of the importance of fast interfacial dynamics of protective surfactants.

\section{Experimental}

\section{Materials}

30 Antibodies: polyclonal human antibody (IgGp) and monoclonal chimeric murine/human antibody (IgGm) were both from LFB Biotechnologies (Laboratoire Français de Fractionnement et des Biotechnologies, France). The Human polyclonal immunoglobulins (IgGp) are prepared from large pools of human 35 plasma and represent the antibody spectrum present in the donor population. Thus, IgGp contains a sampling from the entire array of variable regions of antibodies as well as the IgG subclass distribution expected to be present in normal human serum. IgGp was derived from Human normal Intravenous Immunoglobulin 40 manufacturing (IVIg) as described by the European Pharmacopoeia, Ph. Eur. Monograph 0918. IgGp was produced from fraction I+II+III obtained by cold ethanol fractionation, purified by caprylic acid precipitation followed by chromatography steps and viral inactivation. The resulting IgGp 45 had a purity of $>95 \%$ IgG with trace amount of IgA and IgM. The monoclonal recombinant antibody (IgGm) is a genetically engineered chimeric murine/human monoclonal antibody. It displays the typical structure of immunoglobulin with two gamma heavy chains and two kappa light chains linked with sixteen intra50 and inter-chains disulfide bridges. It is composed of a total of 1,322 amino-acids with molecular weight of $147 \mathrm{kDa}$. Its isoelectric point is around 8.9 with an isoform patern containing five bands between 8.87 and 9.02 . The sample purity (>98\% IgG) was assessed by SDS-PAGE analysis and size exclusion 55 chromatography (HPSEC). Before use both IgGp and IgGm were dialyzed against $36 \mathrm{mM}$ citrate buffer, $\mathrm{pH}$ 6.8, in Slide-A-Lyser dialysis cassette (3500 MWCO, Interchim Thermo Scientific), and the final concentrations were determined by UV absorption measurements using extinction coefficients of 1.4 and $1.61 \mathrm{L.g}-$ ${ }_{60}{ }^{1} . \mathrm{cm}^{-1}$ for IgGp and IgGm respectively. Here citrate buffer meant for a $144 \mathrm{mM}$ sodium dihydrogen citrate- $\mathrm{NaOH}$ solution adjusted to $\mathrm{pH} 6.8$ with concentrated $\mathrm{NaOH}$ and diluted to $36 \mathrm{mM}$ citrate. Deionized water (Millipore, $18 \Omega\left(\mathrm{m}^{-1}\right.$ ) was used for all experiments to rinse, or dilute solutions.

65 Surfactants: Surfactin from Bacillus Subtilis, tertadecyltrimethylammonium bromide (TTAB), dimethyldodecylamine N-oxide (C12NO) and Tween80 (Tw80) were purchased from Sigma-Aldrich; fos-cholines (FC10, FC12 and FC14) were from Affymetrix, lauroylsarcosine sodium salt 70 (LSNa, 30\%wt water solution) was from Fluka. Except LSNa, stock solutions of surfactants were prepared in $36 \mathrm{mM}$ citrate buffer ( $\mathrm{pH}$ 6.8) by dissolving the surfactant dry powder in buffer. Fluorescent anti-human antibody fragments: Rhodamine (TRITC)-conjugated AffiniPure $\mathrm{F}(\mathrm{ab})_{2}$ fragment goat anti75 human IgG, F(ab') ${ }_{2}$ fragment specific (Rhod-antiIgG-Fab) and fluorescein (FITC)-conjugated AffiniPure $\mathrm{F}(\mathrm{ab})_{2}$ fragment goat anti-human IgG, $\mathrm{FC}_{\gamma}$ specific (Fluo-antiIgG-Fc) were purchased from Jackson ImmunoResearch laboratories INC. All other chemicals were from Sigma-Aldrich.

80

\section{Stress-induced IgG aggregation}

$1 \mathrm{~mL}$ aliquots of solutions (1.5 g/L IgGp, or IgGm, with $0.05 \mathrm{mM}$ surfactant or with no surfactant) were filtered through $0.2 \mu \mathrm{m}$ PES WHATMAN syringe filter, and gently introduced (by 85 flowing along the internal wall) into dust-free glass test tubes. The tubes were then closed by a cap wrapped in parafilm and containing an off-centered Sterican ${ }^{\circledR}$ needle (B Braun Medical Ltd.) that was adjusted to penetrate 2-3 mm into the solution (at vertical position). The intensity of scattered light $\left(I_{0}\right)$ was 90 immediately measured by light scattering measurements and the tubes were lied on the roller mixer SRT6D (Stuart ${ }^{\circledR}$ ) positioned at angle $3.5^{\circ}$. Due to its off-centering, the needle penetrates into the solution and pierces the interface at each rotation of the tube (see Figure 1A). Because the diameter of the roller's cylinders 95 and the diameter of the tubes are different, the programmed rotation rates of rollers (45, 20 and $8 \mathrm{rpm})$ correspond to actual rotation rates of the tubes (137, 64 and $22 \mathrm{rpm}$ respectively) which are those indicated in the text and figures. After incubation for a time $t$ under rotation, the intensity of scattered light, $I_{t}$, was 100 measured, and the difference $I_{t}{ }^{-} I_{0}$ is attributed to aggregates. Experiments were done in triplicate for each condition (3 different tubes and needles).

\section{Dynamic light scattering (DLS)}

105 Dynamic laser light scattering was used to detect the aggregates of antibodies. DLS experiments were carried out on a Brookhaven system, equipped with Brookhaven multiple $\tau$ digital correlator, and a laser with an output power of $30 \mathrm{~mW}$, at a wavelength of $637 \mathrm{~nm}$. The data were collected at $90^{\circ}$ angle and $11025^{\circ} \mathrm{C}$. The mean intensity of scattered light was measured over a 1-minute acquisition time. For DLS analysis, the homodyne intensity-intensity correlation function $G(q, t)$ was related to the correlation function of the scattered field where $A$ is the 
experimental baseline and $b$ is the fraction of correlated light. Inversion of the autocorrelation function $g$ was performed using the program NNLS, providing a distribution of apparent Stokes diameters.

\section{Fluorescence Microscopy (FM)}

Approx. $150 \mu \mathrm{L}$ of solution (1.5 g/L IgGp solution in $36 \mathrm{mM}$ citrate buffer pH6.8, or the buffer alone for the blank experiment) was filtered through $0.02 \mu \mathrm{m}$ inorganic membrane syringe filter 10 Anotop10 (Whatman Int. Ltd.) directly into paraffin surface (Parafilm ${ }^{\circledR}$ ) in order to form a sessile drop. Freshly cleaved mica plate (Potassium Aluminosilicate Muscovite Mica from Goodfellow) was then brought into short contact with the drop (approx. 2s) and rinsed immediately twice with $40 \mathrm{~mL} \mathrm{MQ}$ water. 15 The mica slide was then placed on a $100 \mu \mathrm{L}$ filtered drop of $1.5 \%$ BSA solution (in $36 \mathrm{mM}$ citrate buffer pH6.8). After 1.5 hours of incubation in wet atmosphere, $150 \mu \mathrm{L}$ of $1: 1$ mixture of RhodantiIgG-Fab and Fluo-antiIgG-Fc (filtered through $0.02 \mu \mathrm{m}$ syringe filter) was added to BSA solution. After 1.5 hours of 20 incubation in wet atmosphere and in the dark, the mica plate was rinsed twice with $40 \mathrm{~mL} \mathrm{MQ}$ water and stored in water till observations under the microscope.

The mica surface was observed by fluorescence microscopy using a LEICA DM-IRE2 inverted microscope. The fluorescence 25 of Fab specific rhodamine-conjugated Fab fragment goat antihuman IgG (Rhod-antiIgG-Fab) was excited at 515-560 nm and observed at $590 \mathrm{~nm}$, while the fluorescence of the Fc specific fluorescein-conjugated Fab fragment goat anti-human IgG (FluoantiIgG-Fc) was excited at 460-500 nm and observed at 527-560 $30 \mathrm{~nm}$. The signals were recorded on a CoolSnap monochrome camera (Roper Scientific, Germany) under fixed exposure conditions (typical exposure time $3000 \mathrm{~ms}$, binning 2x2).

\section{Atomic Force Microscopy (AFM)}

35 The contact of freshly cleaved mica plate (Potassium Aluminosilicate Muscovite Mica from Goodfellow) with $1.5 \mathrm{~g} / \mathrm{L}$ IgGp solution (in $36 \mathrm{mM}$ citrate buffer $\mathrm{pH6}$.8) was performed in the same way than for fluorescent microscopy sample preparation. After the contact, mica plate was immediately rinsed 40 twice with $40 \mathrm{~mL}$ MQ water and dried at $40^{\circ} \mathrm{C}$. AFM measurements were performed using a 5100 Atomic Force Microscope (Agilent technologies- Molecular Imaging) operated in a dynamic tip deflection mode (Acoustic Alternating Current mode, AAC). All AFM experiments were performed using ${ }_{45}$ Silicon Probes (Applied NanoStructures-FORT) in the tapping mode with spring constant $3 \mathrm{~N} / \mathrm{m}$ at $69 \mathrm{kHz}$. The images were scanned in topography mode with a resolution of 512x512 pixels.

\section{Dynamic surface tension (DST)}

${ }_{50} \mathrm{~A} 1 \mathrm{mM}$ stock solutions of surfactants (or/and respectively $5 \mathrm{~g} / \mathrm{L}$ antibodies, IgGp and IgGm) were prepared at least $24 \mathrm{~h}$ in advance, diluted in $36 \mathrm{mM}$ citrate buffer ( $\mathrm{pH}$ 6.8) to $0.05 \mathrm{mM}$ (resp. $1.5 \mathrm{~g} / \mathrm{L}$ for IgGp and IgGm) and incubated a few hours at $22^{\circ} \mathrm{C}$ prior to measurements on the BPA-1S maximum bubble

55 pressure tensiometer (Sinterface, CAD Instrumentation, France), equipped with a capillary of inner diameter of $0.13 \mathrm{~mm}$. The principle of the measurement is described in ref. 14-16. In brief, the apparatus establishes a stationary regime of air flow and measures the pressure as a function of time. The peak of 60 maximum pressure is reached when the diameter of the air bubble equals the diameter of the capillary, and is followed by further bubble growth and detachment. Laplace equation translates this maximum pressure into a value of surface tension, which is determined for varying air flows, i.e. at varying bubble life times. ${ }_{65}$ The data curves can be analyzed according to models established for simple molecular surfactants. ${ }^{17}$

\section{Results}

\section{Relationship between aggregation and interfacial stress}

Upon shaking, or subjecting to mechanical stress such as a rapid 70 rotation, an aqueous solution of antibodies (either polyclonal, IgGp, or monoclonal, IgGm), one generally observes by naked eye that the solution becomes turbid. ${ }^{18}$ Turbidity betrays aggregation. We used scattered light intensity as an index of aggregation to compare various conditions of stress applied on 75 solutions of IgG. For simplicity, we will first consider polyclonal antibodies IgGp. After filtration through a syringe-filter and gentle introduction in the scattering cell (a dust-free test tube, cf. Experimental section), no aggregates can be detected by dynamic light scattering (DLS) (IgGp concentration of $1.5 \mathrm{~g} / \mathrm{L}$ ). A single 80 peak was observed on the DLS size distribution analysis of samples that were slowly and continuously poured along the wall of the glass test tube (see supporting information, Figure S1).

The stability of IgGp was confirmed by the preservation for days of this single peak and absence of intensity drift in samples 85 that were incubated at rest at $22^{\circ} \mathrm{C}$. The hydrodynamic diameter of $12 \pm 1 \mathrm{~nm}$ corresponds in this case to a monomer of IgGp. In contrast, aggregation can be rapidly detected when various stresses are applied as follows. First we considered dropwise falling down of droplets of the solution from ca. $4 \mathrm{~cm}$ above the 90 interface of the solution in the test tube. Dropwise introduction of half of the total volume $(1 \mathrm{~mL})$ of the sample was carried out four times, with no filtration. The intensity of scattered light following this dropwise re-introduction increases significantly compared to the initial, filtered sample (supporting information, Figure S2). In 95 contrast, similar handling i.e. without filter, but carried out by flowing the liquid in a continuous manner along the tube does not change the intensity of scattered light by more than uncertainty (supporting information, Figure S2). The increase of the intensities of scattered light is thus ascribed to the impact of 100 drops on the solutions, which triggers the formation of aggregates of antibodies.

In order to study the importance of a brief mechanical stress on the surface of the samples, we had recourse to an experimental system enabling better control of the perturbation applied on the 105 air-water interface. This system is schematically represented in the Figure $1 \mathrm{~A}$ by a needle that has been fixed into the cap of the glass tube, so that during continuous rotation around the axis of the tube, the needle crosses regularly the air-water interface (cf. Experimental section for details). First, we checked that this 110 needle-based stress was inducing aggregation of the antibodies. For this purpose, we compared the intensities of light scattered by an IgGp solution that has been subjected to rotation 


\section{A) rotation of tubes with needle:}

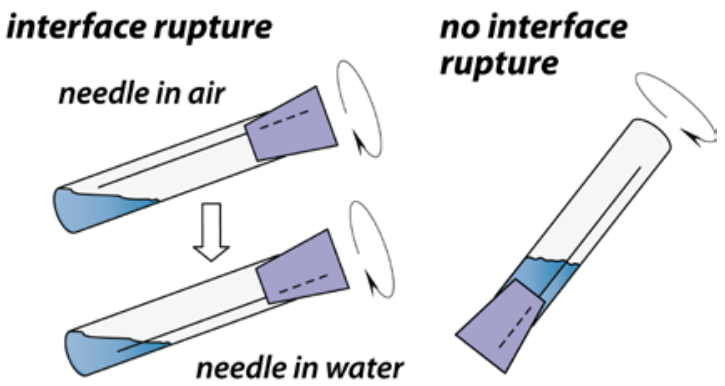

B)

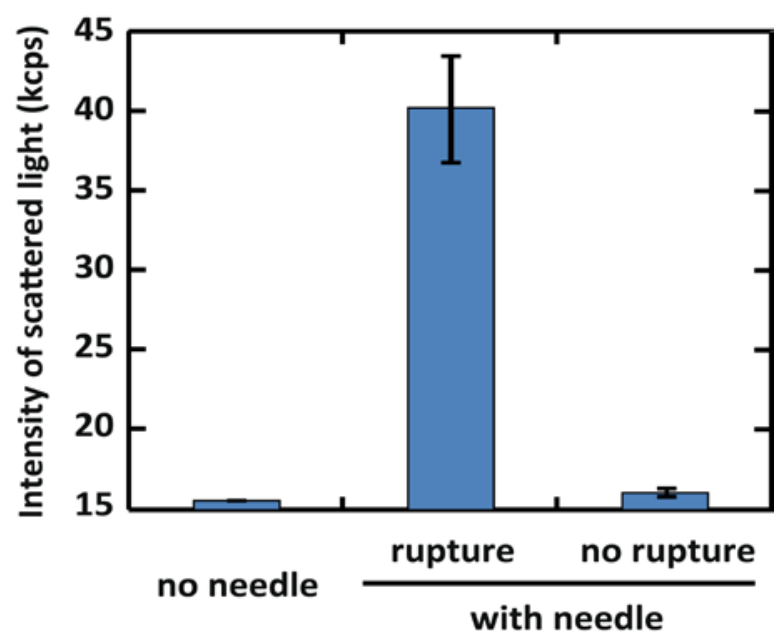

Figure 1. Antibodies aggregation under controlled stress on the air5 water interface of IgG solution. A) Schematic drawing of the experimental system comprising a needle parallel to the axis of the test tube. During rotation of the tube around its axis in tilted position $\left(3.5^{\circ}\right.$ from horizontal), the needle regularly crosses solution-air interface, which is accordingly ruptured (left). When rotation is performed at $45^{\circ}$ angle, 10 the needle stirs the sample and goes up and down through the interface, but remains immersed all the time ("no rupture", right). B) Intensities of scattered light by $1 \mathrm{~mL}$ of $1.5 \mathrm{~g} / \mathrm{L}$ IgGp solution in $36 \mathrm{mM}$ citrate buffer $\mathrm{pH} 6.8, \mathrm{~T}=22^{\circ} \mathrm{C}$ after $85 \mathrm{~min}$. of rotation at $64 \mathrm{rpm}$ with no needle, or with a needle in titled positions $\left(3.5^{\circ}\right.$ or $45^{\circ}$ angles) as quoted by 15 "rupture" and "no rupture” respectively.

of the tubes with and without the needle, for the same time and at the same speed. In presence of the needle, aggregates were clearly detected by an almost tripling of intensity compared to the 20 initial intensity before rotation (Figure 1B).

In contrast, almost no modification of intensity (increase by $<2$ kcps, below the experimental error) occurred in the tubes with no needle, or when the needle was at $45^{\circ}$ angle (i.e. when the needle is kept all the time immersed in the rotating solution). The latter 25 blank experiment shows the absence of aggregation as long as the (stressed) interface is not ruptured, irrespective of stirring of the solution, of contact with needle, and even if the needle moves up and down across the interface. Aggregation only occurred when the needle was alternatively entered in and withdrawn from the 30 solution, across the air-water interface. In practice, rotationtriggered aggregation depended on the geometry of the needle (needle's diameter, angle of penetration), likely because these parameters affects the amount of surface subjected to stress. In the following, we present results obtained with needles having the 35 same size and penetration angle. Under the same condition, repeatability was checked by comparison of several cells having the same geometry: the standard deviation on scattered intensities didn't exceed $13 \%$. This enabled us to reliably study in these simple cells, the importance of rotation speed, time, concentration 40 of antibodies, and presence of surfactants.

\section{Effect of rotation speed and time.}

A quantitative insight on aggregation can be obtained from the study of scattered intensity as a function of rotation speed and ${ }_{45}$ duration. Solutions of IgGp (1.5 g/L, 36mM citrate buffer $\left.\mathrm{pH} 6.8\right)$ were rotated in the presence of needle, at 3 increasing rotation speeds (22, 64 and $137 \mathrm{rpm}$ ), and the intensity of scattered light was regularly measured (by stopping the rotation for ca. 5-8 minutes). The intensity is plotted in Figure 2A as a function of 50 the time of rotation (i.e. leaving out the times needed for measurement of intensity).
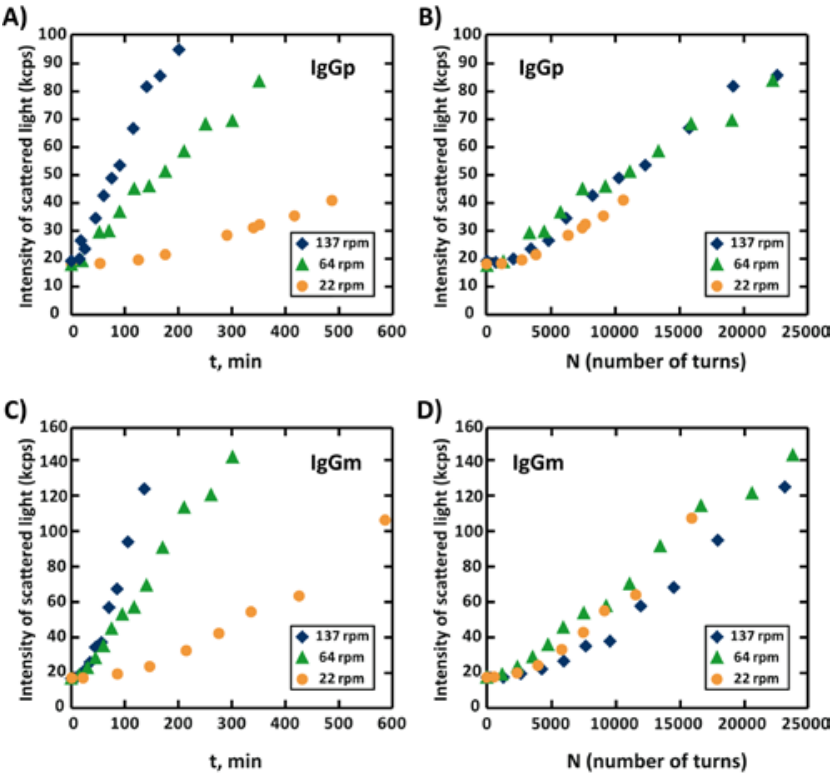

Figure 2. Effect of rotation speed and duration on antibodies aggregation. Scattered intensity by solutions of $\operatorname{IgGp}(\mathrm{A}, \mathrm{B})$ or IgGm $55(\mathrm{C}, \mathrm{D})$ as a function of the total duration of rotation $(\mathrm{A}, \mathrm{C})$ or total number of turns, N (B,D). Experiments were conducted at rotation speeds of: 22, 64 or $137 \mathrm{rpm}$ as quoted. [IgG] = $1.5 \mathrm{~g} / \mathrm{L} ; 36 \mathrm{mM}$ citrate buffer, $\mathrm{pH} 6.8$; T $=22^{\circ} \mathrm{C}$.

The intensity of scattered light increases with increasing time, 60 and a significant part of the data shows a linear dependency. The higher are the rotation speeds, the higher are the slopes in Figure 2A, which may suggest faster aggregation at higher stresses. However, normalization of the abscissa by the number of turns in the Figure 2B gathers all the data into a single master curve. ${ }_{65}$ Regardless of the rotation speed, the aggregation of antibodies is therefore essentially controlled by the number of turns, which is proportional to the number of penetrations of the needle through the air-solution interface. Altogether, the above data show that the number of brief mechanical ruptures of the interface ${ }_{70}$ dominates the appearance of aggregates in solution. 


\section{Case of monoclonal IgG (IgGm)}

In order to show that the interfacial stress-induced aggregation of antibodies was not restricted to IgGp, we studied similarly the effect of rotation with needle on solutions of a monoclonal IgGm.

5 Results on the intensity of light scattered by $1.5 \mathrm{~g} / \mathrm{L} \mathrm{IgGm} \mathrm{in}$ $36 \mathrm{mM}$ citrate buffer $\mathrm{pH} 6.8$ are given in Figure 2C. The rotation in presence of alternating penetration/withdrawal of a needle induces the expected increase of the intensity with increasing number of interfacial piercing. As for IgGp, we found also that 10 data superimpose upon normalization by the number of turns, and that intensity increases linearly as function of rotation time regardless of the rotation rate. We also checked that the apparent hydrodynamic diameters of the native IgGm monomer and aggregates (as estimated by DLS) were in the same range as those 15 found for IgGp.

\section{Absence of growth in the bulk.}

We took into consideration that aggregates, although they are clearly nucleated upon perturbation of the interface, may also

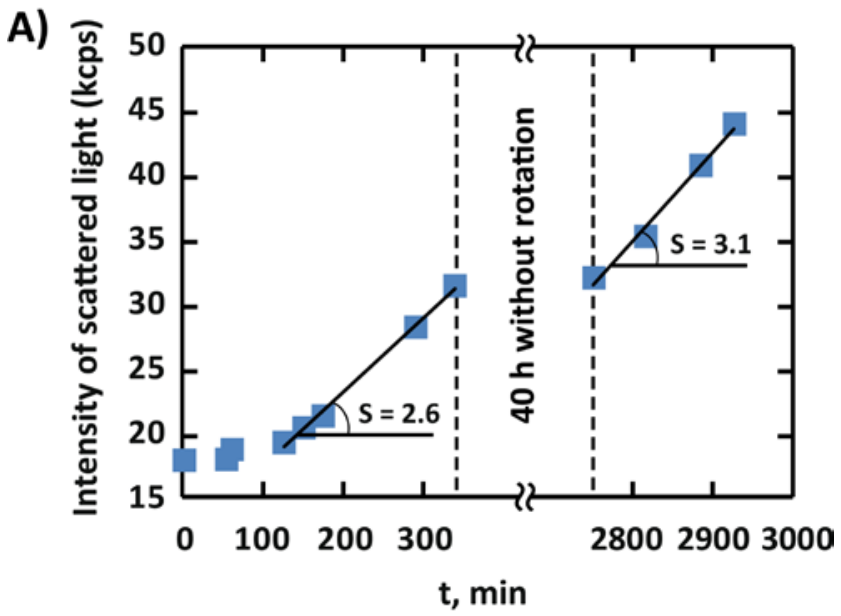

B)

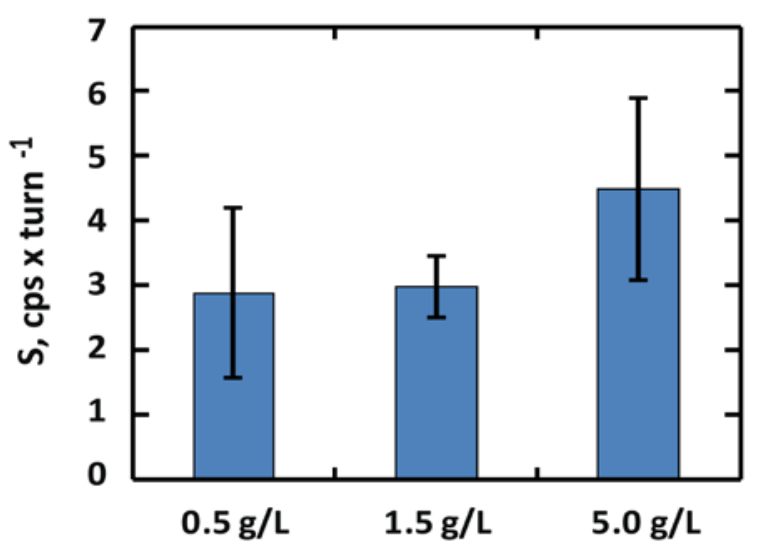

20 Figure 3. Rates of rotation-induced aggregation of IgGp. A) Before and after a 40 hours break of the rotation (22 rpm with needle) that enables the incubation of aggregates in the bulk. "t" is the absolute time value of the experiment (from the beginning of rotation). Values quoted " $\mathrm{S}=$ " in the figure give the slope of linear variation of scattered intensity 25 (unit: cps turn-1). B) Slopes "S" as obtained for experiments of rotation at $64 \mathrm{rpm}$ with needle, at increasing IgGp concentrations (0.5, 1.5 and 5 $\mathrm{g} / \mathrm{L}$ ). Error bars show the standard deviations observed on three replicates. All experiments were performed in $36 \mathrm{mM}$ citrate buffer $\mathrm{pH}$ $6.8 ; \mathrm{T}=22^{\circ} \mathrm{C}$.
30 growth either on the interface or in the bulk. In order to detect a possible contribution of IgG from the bulk, we performed experiments at various IgGp concentrations and we added delays as described below. In a representative experiment, a solution of IgGp $(1.5 \mathrm{~g} / \mathrm{L})$ was subjected to a first rotational stress (with 35 needle) at $22 \mathrm{rpm}$ for $340 \mathrm{~min}$., which induces as expected the gradual increase of the intensity of scattered light with increasing duration of the rotation (Figure 3A). Rotation was stopped (first vertical dashed line in figure 3A), and the tube was left at rest, here for 40 hours. In absence of rotation, the scattered intensity 40 remained unchanged $(\mathrm{I}=32.1 \mathrm{kcps}$ in figure 3A). Rotation was then resumed (second vertical dashed line), at the same speed as the initial one. The slopes, S, of the variation of intensity vs number of turns in figure $3 \mathrm{~A}$ point to a remarkable similarity of the aggregation rate before and after the incubation at rest (3.1 $45 \mathrm{cps}$ turn $^{-1}$ vs $2.6 \mathrm{cps} \operatorname{turn}^{-1}$ ). Thus we conclude on the lack of contribution to aggregation rate of incubation in the bulk.

Finally, we compared needle-induced aggregation at the same rotation rate $(64 \mathrm{rpm})$ but at three different IgGp concentrations $(0.5,1.5$ and $5.0 \mathrm{~g} / \mathrm{L})$. Figure 3B shows the slopes, S, 50 representative of the rate of variation of the intensity of scattered light as function of number of turns. Over one decade range of bulk concentrations, we got a maximum of 1.6-fold increase of the slope $S$ (from $2.8 \pm 1.3$ to $4.5 \pm 1.4 \mathrm{cps}_{\text {turn }}{ }^{-1}$ ). The variation was actually of the order of magnitude of the experimental error 55 (estimated by standard deviation of experiments made in triplicate). The bulk IgG concentration has accordingly a minor role on the observed aggregation.

\section{Visualization of IgG aggregates}

We observed IgG aggregates immobilized on mica by fluorescent 60 microscopy (FM). For this purpose, two fluorescent markers were used: Fab specific rhodamine-conjugated Fab fragment goat antihuman IgG (Rhod-antiIgG-Fab), and Fc specific fluoresceinconjugated Fab fragment goat anti-human IgG (Fluo-antiIgG-Fc). The experiments were performed as follows. A drop of freshly ${ }_{65}$ filtered (through ANOTOP $0.02 \mu \mathrm{m}$ syringe filter) IgGp solution was deposited on parafilm. Freshly cleaved mica plate was brought into brief contact with the apex of the drop (approx. 2s). The mica slide was then extensively rinsed with water and incubated in $1.5 \%$ BSA solution for 1.5 hours. To specifically

70 label IgG adsorbed on the mica, anti-IgG fluorescent probes were then added to the BSA solution and the mica slide was incubated for an additional 1.5 hours (in the dark). After rinsing the slides, the fluorescent microscopy observations were performed using blue/green excitation/emission filters for fluorescein detection 75 and green/red filters for rhodamine detection (see Experimental section for more information). Figure 4A shows representative FM images obtained by double labeling using a 1:1 molar mixture of Rhod-antiIgGh-Fab and Fluo-antiIgGh-Fc. One can see that in absence of IgGp in the drop ("-IgGp” in the figure), a 80 weak, almost homogeneous background is observed on the mica surface both with Rhod-antiIgG-Fab (left) and Fluo-antiIgG-Fc (right) detections (fluorescence intensities: 130.4 \pm 1.4 and 115.2 \pm 1.1 respectively). After contact with a drop containing 1.5 g/L IgGp ("+IgGp”), the fluorescence intensity of the background 85 is significantly higher ( $545 \pm 24$ and $235 \pm 26$ for rhodamine and fluorescein detection respectively) and in addition, submicron and up to micron wide spots are revealed. 
A)
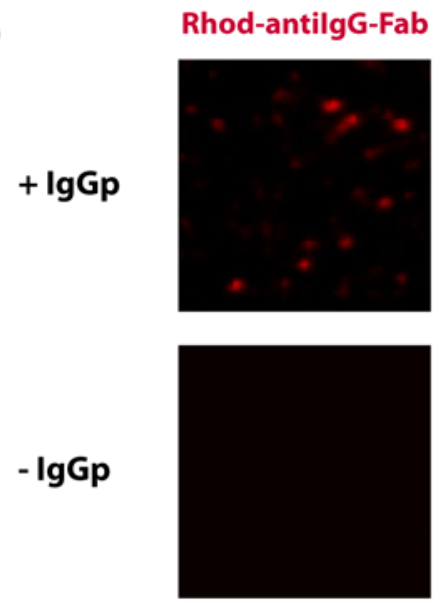

B)

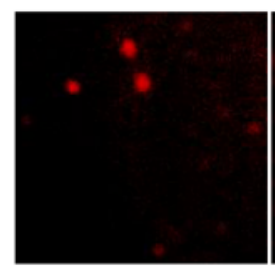

$3 \mathrm{~s}$

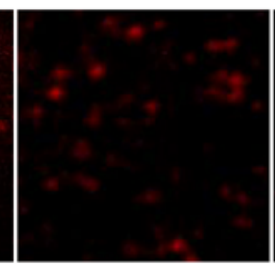

$1 \mathrm{~min}$
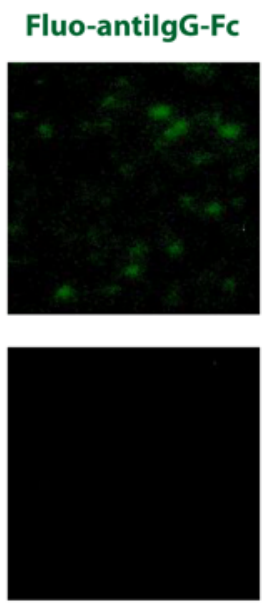

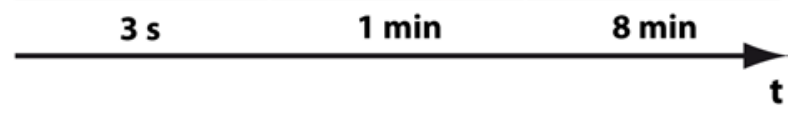

Figure 4. Fluorescent microscopy observation of IgGp immobilized on freshly cleaved mica by a brief horizontal contact with the airsolution interface. Fluorescent microscopy images were obtained after 5 extensive rinsing of the micas with water followed by incubation with anti-IgG markers (a 1:1 molar mixture of Rhod-antilgG-Fab and FluoantiIgG-Fc). (A) Left column corresponds to excitation at 515-560 nm and emission recorded at $590 \mathrm{~nm}$ (rhodamine fluorophore), and right column corresponds to the same position with excitation at $460-500 \mathrm{~nm}$ 10 and observation filter of 527-560 nm (fluorescein fluorophore). "+IgGp" is a mica plate that was deposited on a solution of $1.5 \mathrm{~g} / \mathrm{IgGp}$, “-IgGp" is a reference deposited on a buffer with no IgGp. (B) Mica deposited on drops of increasing age (i.e. incubation time after drop formation) and labeled as for (A). All images are squares of $15 \mu \mathrm{m} \times 15 \mu \mathrm{m}$.

${ }_{15}$ For example, in the case of rhodamine detection, the presence of spots leads to $11.2 \%$ (from $545 \pm 24$ to $606 \pm 11$ ) increase of the average fluorescence intensity measured on 9x9 $\mu \mathrm{m}$ squares). These fluorescent spots were essentially present in the center of the mica, i.e. in the location of the initial contact with the drop, 20 whereas close to the vertices of the mica sheet, we hardly saw heterogeneities in the fluorescence background. Interestingly, the Fab- and Fc- specific markers reveal absolutely the same pattern, which suggests that the marked spots display IgG with both Fab and Fc regions accessible. Anti-IgG labeling with either 25 fluorescent anti-Fab or anti-Fc leaded to similar FM images, though only one color was determined (data not shown).

Altogether, these results point to the detection of i) a background of IgG adsorbed on the mica, and ii) submicron and micron heterogeneities in the density of IgG, likely to reflect 30 aggregates present on the surface of the drop. We also considered the effect of drop age. For this purpose the contact of the mica plate with the sessile drop was delayed by $1 \mathrm{~min}$. or $8 \mathrm{~min}$. after the deposition of the just-filtered drop on the parafilm. The images presented in the Figure $4 \mathrm{~B}$ show the highest density of 35 fluorescent spots found by random exploration of the mica

\section{A)}

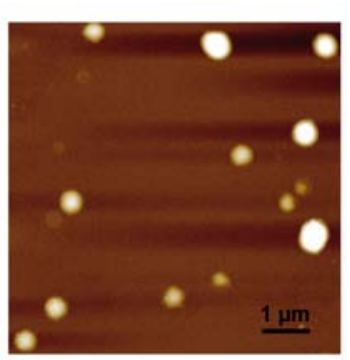

B)

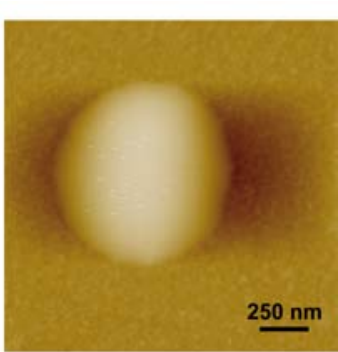

C)

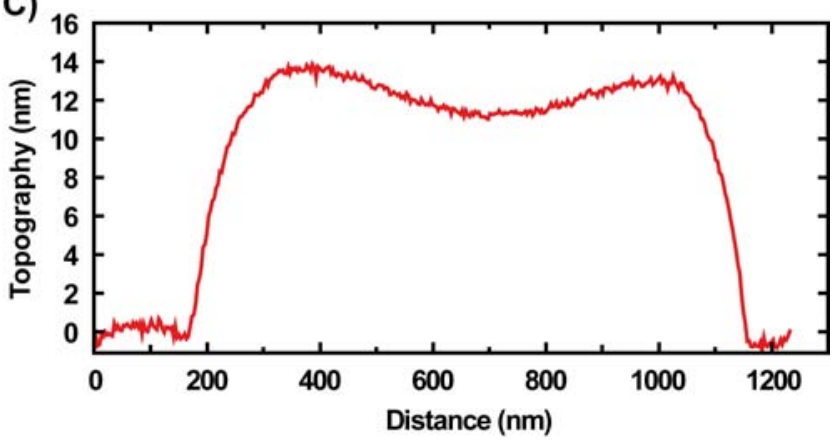

Figure 5. AFM observation of IgGp aggregates immobilized on freshly cleaved mica by a brief horizontal contact with the air-solution 40 interface. AFM images were obtained after extensive rinsing of the mica with water followed by drying at $40^{\circ} \mathrm{C}$. A) Typical AFM image showing submicron and up to micron size objects; B) Typical IgGp aggregate, and C) Topographic profile of an IgGp aggregate of $950 \mathrm{~nm}$ diameter and 12$14 \mathrm{~nm}$ height.

45

surface. Few aggregates are formed at the shortest times of ca. 3s. The number of spots increases significantly with increasing time.

Mica-immobilized aggregates were in addition characterized by atomic force microscopy (AFM), at long age of the drop 50 (approx. 5 min., to increase the probability of observation of aggregates). Deposition procedure was the same to that used for FM, but with no labeling step: immediately after the rapid contact of the drop with the mica, the plate was rinsed with excess of water, and dried at $40^{\circ} \mathrm{C}$. Representative objects observed on the 55 mica's surface are shown in Figure 5 . One can observe the presence of submicron and up to micron wide spots (Figure 5A,B). Moreover, these objects were essentially observed in the location of the initial contact with the drop. An example of the topographic profile of a representative object is shown in Figure 60 5C. Typically, the width of such objects varies between $200 \mathrm{~nm}$ and $1200 \mathrm{~nm}$, and their height corresponds to the diameter of an individual antibody molecule (12-14 nm).

Of importance to confirm the origin of the background fluorescence in FM, we found that the mica was covered by a 65 monolayer of IgG. In a region devoid of aggregates, we subjected the surface to a "scratch" by applying a high force of $200 \mathrm{pN}$, while scanning. This procedure is expected to displace weakly adhesive substrates bound on the mica. Accordingly, an image scanned in milder conditions just after the scratch revealed 70 (supporting information, Figure S3) that the stressed region displays a lower height by $-11 \mathrm{~nm}-13 \mathrm{~nm}$ compared to background. The loss of height compares with the size of one IgG molecule, which is in agreement with the expectation of a 
random, though dense, adsorption on the mica of monomers of the protein. As extensively studied, though with other proteins, the diffusion from the bulk of the solution provides proteins that bind tightly on the freshly cleaved mica ${ }^{19,20}$ and eventually form

5 a monolayer. Aggregates are thus detected above the adsorbed monolayer of IgG.

\section{Protection by surfactants}

To investigate the origin of protection brought by surfactants, we compared solutions of IgGp at a fixed concentration $(1.5 \mathrm{~g} / \mathrm{L})$, in 10 presence of a fixed concentration of various surfactants $(0.05$ $\mathrm{mM})$. These concentrations are representative order of magnitudes of the concentrations used to stabilize biotherapeutic formulae. We used surfactants having obviously different polar heads and of varying hydrophobic chain lengths, in order to study 15 a wide variety of compounds likely to differ in term of interactions in bulk and at interface (e.g. affinity between IgG and surfactants, competition for adsorption, etc). Accordingly, we selected a library of widely used surfactants: Tween80 (also called Polysorbate 80 and abbreviated as Tw80), fos-cholines 20 whose hydrophobic tail length was varied from 10 to 16 carbon atoms (FC10-FC16), dimethyldodecylamine N-oxide (C12NO), lauroylsarcosine sodium salt (LSNa), tetradecyltrimethylammonium bromide (TTAB), and a natural surfactant from Bacillus subtilis, surfactin, bearing a peptidic head group. This 25 library contains neutral, zwitter-ionic, cationic and anionic surfactants, with aliphatic tails of length comprised between 10 and 18 carbon atoms.

In presence of any surfactant from the list above (at $0.05 \mathrm{mM}$ ) no aggregation of IgGp could be detected by light scattering after ${ }_{30} 6 \mathrm{~h}$ of rotation (rotation at $3.5^{\circ}$ angle, with needle, $64 \mathrm{rpm}$, data not shown). This obviously confirms that all the surfactants efficiently slow down the aggregation of IgGp. In order to be able to compare their relative efficiencies, we chose the following harsher conditions: $65 \mathrm{~h}$ of rotation with needle at $64 \mathrm{rpm}$, ${ }_{35} \mathrm{~T}=22^{\circ} \mathrm{C}$. The results presented in Figure $6 \mathrm{~A}$ give values of intensity difference, $\left(I_{65 h}-I_{0}\right)$, i.e. the excess intensity of scattered light due to the presence of aggregates. This enabled us to determine the order of efficiencies of surfactants as inhibitors of IgGp aggregation: surfactin $>$ Tw80 $>$ TTAB $>$ FC12 $\approx$ C12NO $>$ $40 \mathrm{LSNa}$. In the case of a homologous set of fos-choline surfactants, the efficiency lists in the order FC14 > FC12 > FC10 (Figure 6B). Surface tension has been extensively used to study adsorption and interaction taking place at the air-water surface in surfactant/protein mixtures. ${ }^{21,22-25}$

45 Of importance with regards to the short (minutes) time scale of aggregation of antibodies on surface (cf. above), maximum bubble pressure (also called Dynamic Surface Tension measurement, DST) enabled us to record the surface tension variations at short times after creation of air-water interface, over

50 time window ranging from ca. $10 \mathrm{~ms}$ to $100 \mathrm{~s}$. DST curves are indicative of the kinetics of adsorption of molecules, which can be translated in quantitative terms by equation 1 in the limit of short times. As illustrated in Figure 7, the DST curves of our set of surfactants, at the fixed concentration of $0.05 \mathrm{mM}$, cover more

55 than a decade of adsorption rates. In Figure 7A, the curves can be ranked in the order of increasing times at which a threshold value of surface tension is reached. Using somewhat arbitrarily a value of $66 \mathrm{mN} / \mathrm{m}$, surfactants rank as follows: surfactin > Tw80 >
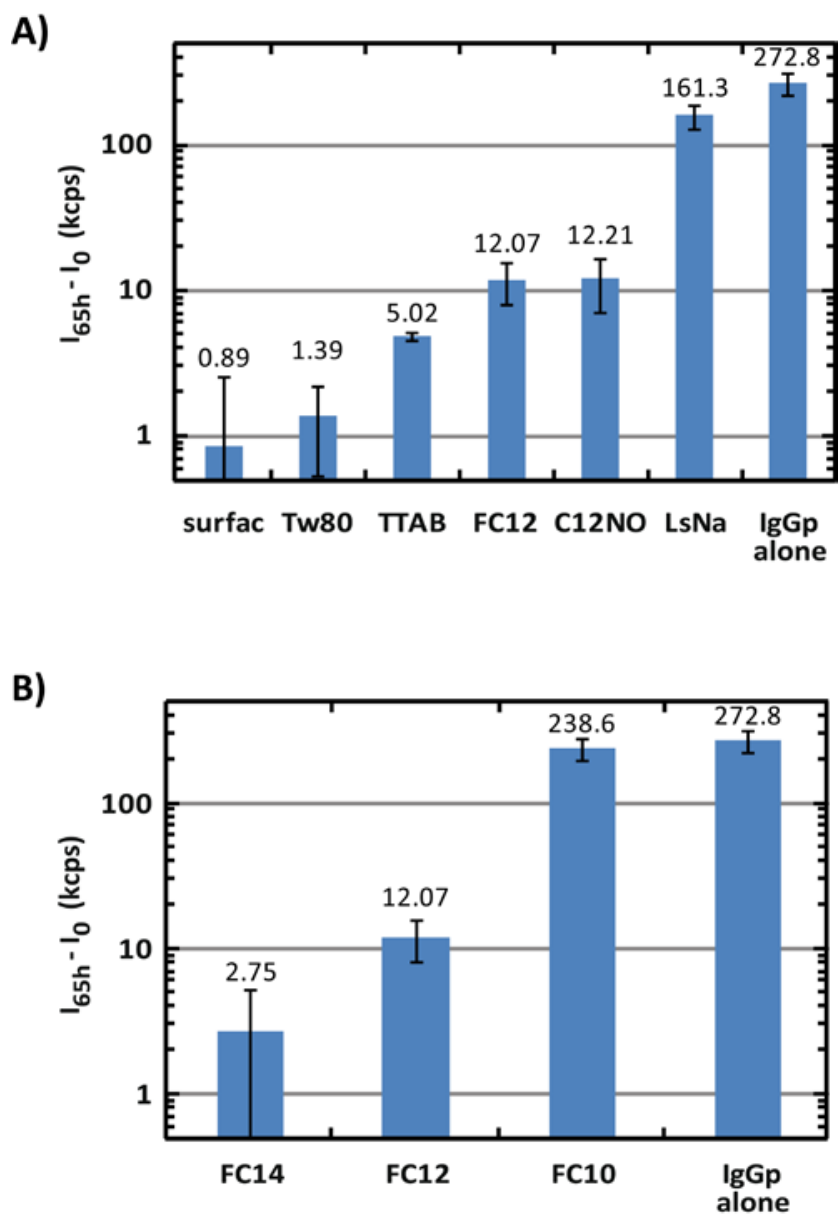

${ }_{60}$ Figure 6. Inhibition of interfacial stress-induced IgGp aggregation in the presence of surfactants. Excess scattering intensity $\left(\mathrm{I}_{65 \mathrm{~h}}-\mathrm{I}_{0}\right)$ by IgGp solutions $(1.5 \mathrm{~g} / \mathrm{L})$ is determined after 65 hours of rotation with needle in the presence of $0.05 \mathrm{mM}$ surfactants in $36 \mathrm{mM}$ citrate buffer $\mathrm{pH} 6.8$; $\mathrm{T}=$ $22^{\circ} \mathrm{C}$. (A) Set of surfactants having varying nature of head groups; (B) 65 Fos-cholines of varying lengths of the tail; In the X-axis, "surfac" meant for surfactin. Error bars show the standard deviations observed on three replicates. With surfactin no variations of intensity was detected in 2 samples, and the third one was more turbid, which increased considerably the estimated error. Quotes above the bars are the values of excess 70 intensities equal to intensity at time $65 \mathrm{~h}$ minus initial one.

$\mathrm{TTAB}>\mathrm{FC} 12 \approx \mathrm{C} 12 \mathrm{NO}>\mathrm{LSNa}$. And within a homologous set of fos- cholines (Figure 7B), the rate of adsorption decreases with decreasing length of the tail: FC14 > FC12 > FC10 (FC16 was not completely soluble at the test concentration, and it was finally 75 not used). The rate of decrease of surface tension thus correlates with the protection efficiency of the surfactants.

In absence of energy barrier against adsorption, which is a reasonable expectation at low surface coverage, the surface tension reflects adsorption that should be limited by diffusion. ${ }_{80}$ For molecular surfactants, it is expected that surface tension varies in proportion to the square root of time (equation 1$)^{14}$ :

$$
\gamma(t)_{t \rightarrow 0}=\gamma_{0}-2 n R T C \sqrt{\frac{D t}{\pi}} \quad \text { Eq. } 1
$$

With $C$ the molar concentration of the surfactant, $n=1$ for neutral or 2 for ionic surfactants, $D$ the apparent diffusion coefficient of surfactant toward interface, $R$ Boltzmann constant, $T$ temperature, 

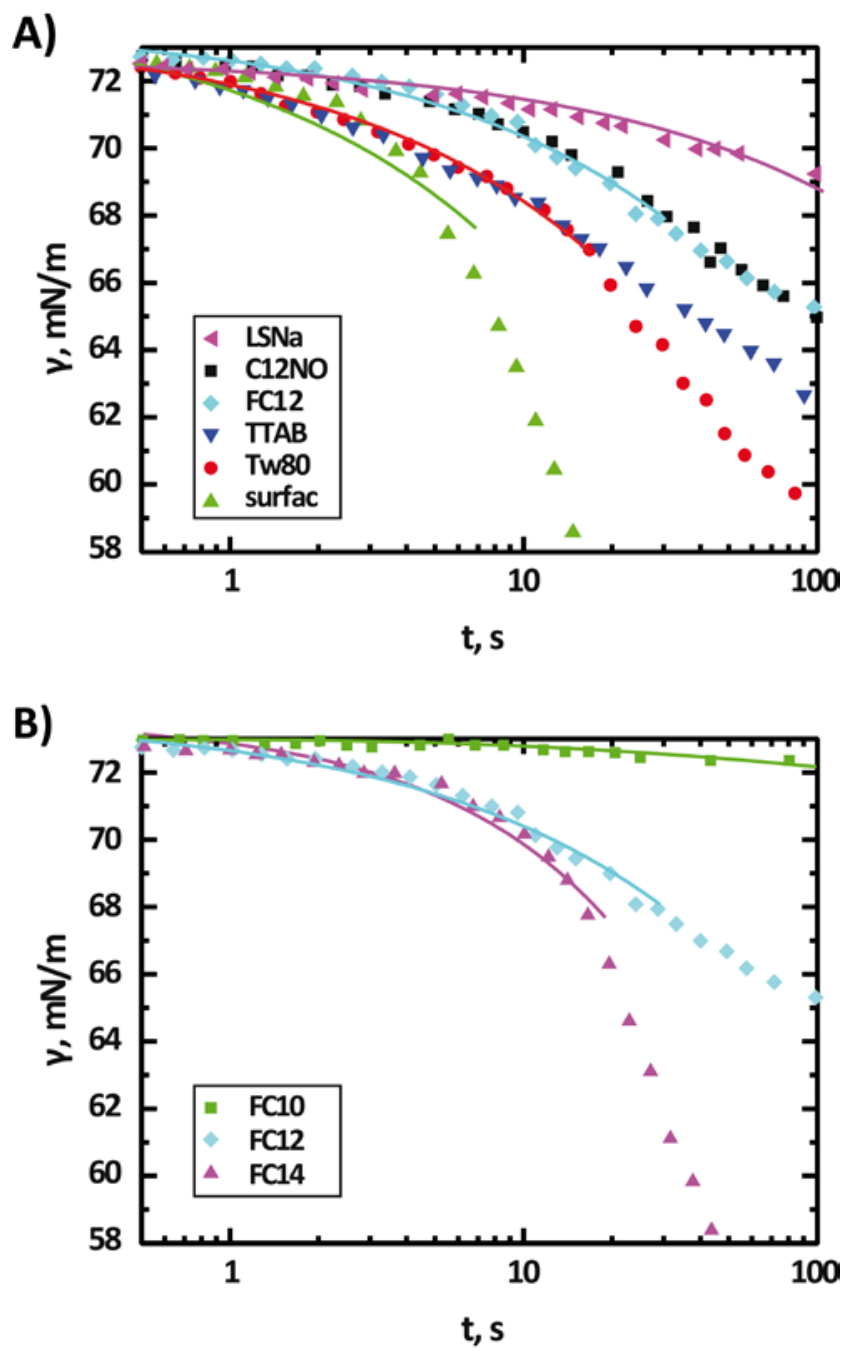

Figure 7. Dynamic surface tension of solutions of surfactants at $\mathbf{0 . 0 5}$ mM. Measured by maximum bubble pressure in $36 \mathrm{mM}$ citrate buffer $\mathrm{pH}$ 6.8, $\mathrm{T}=22^{\circ} \mathrm{C}$ as function of the time of life of bubbles. (A) Set of 5 surfactants having varying nature of head groups; (B) Fos-cholines of varying lengths of the tail. Lines show fits by eq.1. The acronyms of surfactant are given in the figure and defined in the text; "surfac" meant for surfactin.

10 and $t$ the age of the interface. Except for surfactin, good matching of the data with equation 1 was obtained in the limit of short time scale (Figure 7). When they are listed in order of decreasing D, neutral surfactants follow the same order as above. But due to the contribution of counter ions in equation $1(n=2)$, the apparent 15 diffusion coefficient of ionic surfactants having similar DST curve is calculated to be four-time lower than those of a neutral surfactant. Accordingly, the dynamics of adsorption of surfactants on freshly created interface is listed as follows: surfactin $>$ Tw $80>$ FC12 $\approx$ C12NO $>$ TTAB $>$ LSNa. Efficiency 20 of protection brought by the various surfactants correlates well with kinetics of surface tension decrease, but not with the dynamics of adsorption when ionic surfactants are considered (in absence of proteins).

In mixtures of protein and surfactant, surface tension could be 25 affected by contributions of both species, and possibly reveals interactions between IgG and surfactants. Impact of lateral

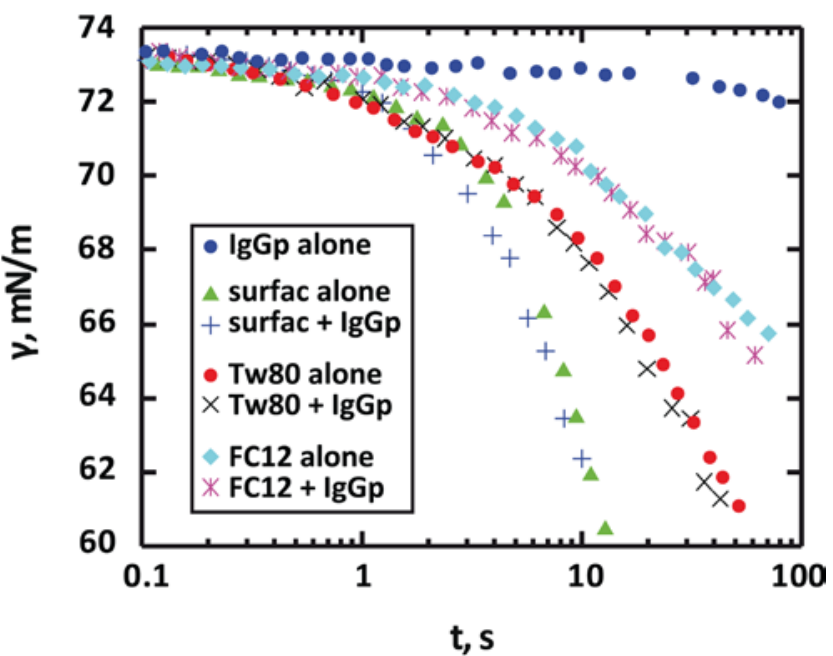

Figure 8. Comparison of dynamic surface tension curves of surfactants in the presence and absence of IgGp. Measured by 30 maximum bubble pressure in $36 \mathrm{mM}$ citrate buffer $\mathrm{pH} 6.8, \mathrm{~T}=22^{\circ} \mathrm{C}$ as function of the time of life of bubbles. Surfactants concentration was 0.05 $\mathrm{mM}$. When used, $[\mathrm{IgGp}]=1.5 \mathrm{~g} / \mathrm{L}$. The acronyms of surfactant are given in the figure and defined in the text; "surfac" meant for surfactin.

35 interaction on the adsorption isotherms has been recently formalized by Miller and coll. ${ }^{21}$ At times as short as a few seconds, one expect in the present experimental condition that IgG can cover a significant fraction of the inrterface. A characteristic time for surface coverage by IgG was estimated by

${ }_{40}$ DST measurement on IgG solutions with no surfactants (Figure 8 and Supporting information Figure S4). At $1.5 \mathrm{~g} / \mathrm{L}$ IgGp and IgGm (the concentration used throughout this study), the onset of surface tension decrease occurs beyond a time of approximately 20s-30s. According to Gurkov et al., ${ }^{26}$ one expects that the time 45 of abrupt decrease of the surface tension in a solution of proteins corresponds to reaching a surface coverage by proteins of the order of $50 \%$. Representative DST measurements on mixtures of IgG and surfactants are presented in Figure 8. Within experimental error, the values of surface tension in presence of 50 IgG superimpose with values in the absence of protein for all neutral surfactants tested. In the whole time window $(t<100 \mathrm{~s})$, surface tension is thus dominated by the contribution of the surfactant (both with IgGp and IgGm and for all neutral surfactants, data not shown). This lack of contribution of the 55 protein suggests that interactions with surfactants are essentially negligible on the interface. Binding isotherms as extensively analyzed by Miller and coll. ${ }^{21}$ contain terms accounting for the formation of complexes between adsorbed surfactants and proteins. Several complicated effects may be competing, 60 including a variable specific area per protein (which depends on orientation of the protein at the surface), entropy terms due to the asymmetry of size of surfactant vs protein, and energy due to lateral interaction. If the surface excess of protein increases with time, up to ca $50 \%$ coverage, a perfect compensation of all terms 65 in the whole time window require is highly unlikely. Results of DST measurements thus indicate that either IgG are not significantly adsorbed in presence of surfactants (including well before surface saturation), or that their interactions with neutral surfactants on the interface are negligible. The hypothesis of a 
barrier against IgG adsorption however does not match with observations. In presence of surfactin, FM displayed few, though obvious, spots (supporting information, Figure S5). Similarly, we observed by AFM the presence of aggregates, in Tw80 protected 5 samples of IgGm (data not shown). Despite the presence of a highly protective surfactant, some aggregates were still present on the surface of drops.

\section{Discussion}

\section{Aggregation of antibodies occurs at the interface}

${ }_{10}$ Various external factors able to induce aggregation of proteins are described in the literature, including temperature, ${ }^{27,}{ }^{28} \mathrm{pH}^{28}$ ionic strength, ${ }^{29}$ freeze-thawing, ${ }^{30,31}$ shearing, $^{32}$ stirring $^{13,18}$ and shaking. ${ }^{18,33-35}$ Because one can readily manage with composition and temperature parameters, most proteins can now be made

15 highly stable under the form of liquid preparations at rest. In contrast, mechanical stresses are hardly avoidable while filling, handling flasks. In the present investigation we bring evidences of an early step of aggregation, likely to be involved in conditions of stress: the formation of IgG aggregates upon transient 20 breakage of air-water interface. The interface, even in absence of shear in the case of a sessile drop, is shown by FM to contain an increasing amount of aggregated IgGp with increasing incubation time. Aggregates were recognized by their height $(12-14 \mathrm{~nm}$ by AFM) and heterogeneities in fluorescence after specific labeling 25 (anti-Fab and/or anti-Fc in FM). They formed at the interface within less than one minute. Therefore "primary" surface aggregates stay at the interface and don't penetrate into the bulk in absence of external triggers. This was confirmed by two experimental observations: NO aggregates are detected in the 30 solution, neither after mild introduction and slow flow of the solution of IgG along the wall of light scattering cells, nor after rotation of solutions in absence of mechanical stress applied on the air-water interface (rotation without needle). In addition, the lack of contribution of bulk concentration of IgGp to the growth 35 rate of aggregates and the variation of scattered intensity in proportion to the number of interfacial breakage, both suggest the predominant role of surface on the nucleation and growth of aggregates. A schematic representation of the proposed mechanism of aggregation is present in the Figure 9.

40

Which characteristic properties of interface correlate with prevention of aggregation?

Conventional analysis of the protection brought by surfactants is based on studies at equilibrium and on the idea of competitive 45 hydrophobic adsorption. In presence of surfactants, equilibrium adsorption displaces the adsorbed proteins. ${ }^{22-25,36}$ Although we do not deny the capacity of the surfactants with higher surface activity to displace antibodies from the interface, our results show however the presence of aggregates at the surface, even in the 50 presence of surfactants (surfactin, Tw80). Surfactants used here at concentrations $0.01 \mathrm{mM}$ to $1 \mathrm{mM}$ reach an important degree of surface coverage within a few minutes, i.e. at times rather short compared to incubation times of samples in our conditions: accordingly, one expect that they would protect the interface if 55 competitive displacement were efficient against aggregation. In addition, our results point to the lack of contribution of IgG bulk
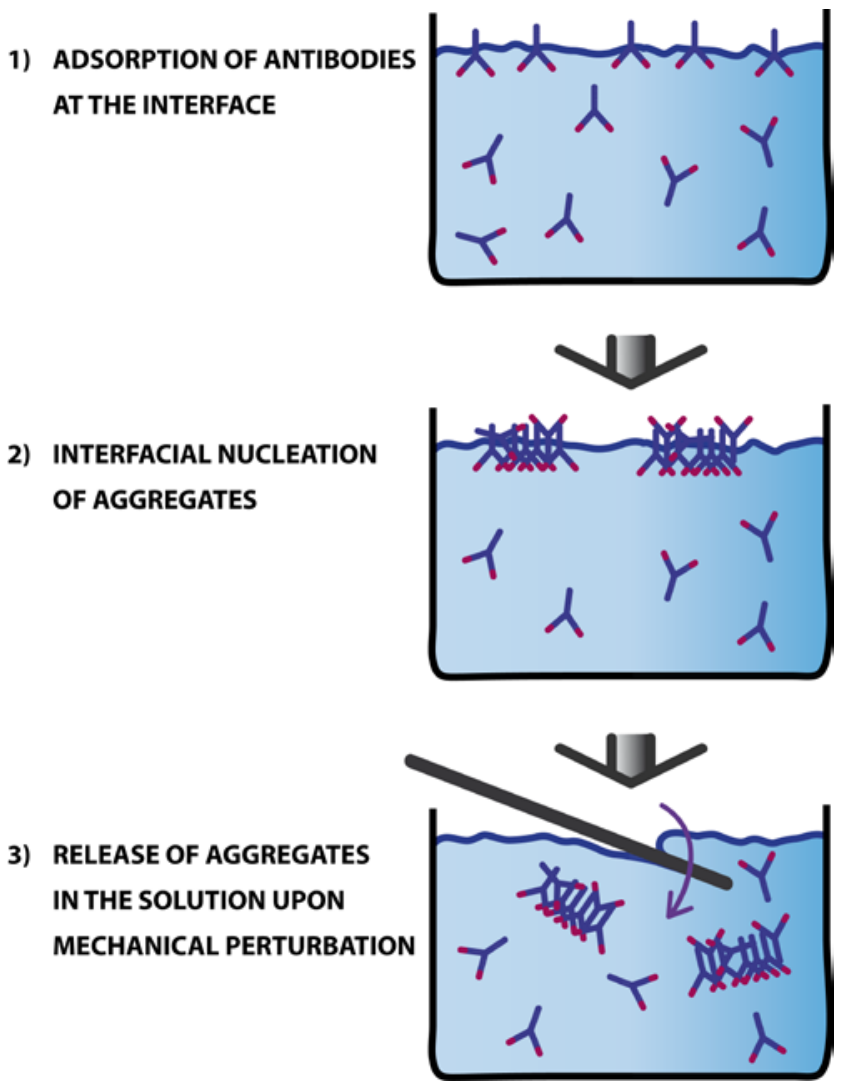

Figure 9. Schematic drawing of interfacial stress-induced IgG aggregation. The nucleation of aggregates and their growth takes place 60 essentially at solution-air interface $(1,2)$ and aggregates stick stable on the surface until (3) their release into the bulk occurs upon mechanical perturbation of the interface.

concentration on the kinetics of aggregation which is difficult to understand in the framework of a displacement by surfactants or a ${ }_{65}$ barrier against adsorption. Interestingly also, being above or below critical micellar concentration does not make any difference in the protection (see supporting information, Table S1). All these observations indicate therefore that displacement of adsorbed antibodies at equilibrium with surfactants shall occur 70 but is not sufficient to explain the protection against mechanical stress. On the other hand, it is well established that cationic surfactants induce aggregation of (anionic) proteins in the bulk. ${ }^{37,38}$ Our observation of protection of IgG in presence of the cationic surfactant, TTAB, strengthen the idea that association 75 and/or aggregation in solution is not the main origin of destabilization of IgGs.

Altogether, our results finally demonstrate that protection occurs from the lack of transfer of the aggregates from surface into the solution, and in the presence of aggregation on interface. ${ }_{80}$ Our results also suggest that the kinetics of decrease of surface tension by surfactants is indicative of the efficiency of protection against aggregation: protection is observed with surfactants that can significantly decrease the surface tension before a significant fraction of the surface is covered by the protein. In practice, 85 however, the stresses were applied on an interface whose age can be as long as the incubation time of the solution. Even the time zero (onset of rotation) corresponded to a several-minute long incubation at rest, prior to rotating the tubes. Under these conditions, it appears rather surprising that kinetics of adsorption 
may contribute to modulate aggregation. We suggest that the correlation between protection and the values of surface tension reached at short time scales should be rooted in a contribution of the interfacial energy stored during the stretching of interface and 5 released upon breakage of the film. A driving force is required for the transfer of aggregates in solution. Lower surface tension implies lower energy stored in the interface. When surface stretch is more rapid than the dynamics of surfactant needed to equilibrate tension variations, the energy released by the rupture 10 of the film should be maximized. In addition, competitive adsorption and diffusion of surfactant and IgG governs the composition of freshly born interfaces formed under the stretching conditions applied by the needle. Obviously the rupture of a film that would contain primarily surfactants would 15 contribute to lesser extent to the release of IgG aggregates. Finally, we propose two tentative origins of protection: both related to transient phenomena occurred in freshly stretched airwater films: i) rapid lowering of the energy of stretched films, and ii) rapid adsorption or diffusion of surfactants into the film 20 compared to proteins. Both mechanisms basically rely on a dynamics of surfactants that must be more rapid than the applied stress.

\section{Conclusions}

By using a controlled perturbation of air-water interface, we 25 showed that the IgG aggregates appear in solutions of stable antibodies because of interfacial stress. The amount of aggregates in solution (determined by light scattering) varies in proportion to the number of times the interface is mechanically perturbed, irrespective of the nature and concentration of IgG. Primary 30 aggregates were characterized by AFM, on freshly cleaved mica briefly brought into contact with the surface of IgG solution. They appear under the form of oblate objects with diameter $200 \mathrm{~nm}-1200 \mathrm{~nm}$ and height of the order of once or twice the protein diameter. Specific antibody labeling, using anti-Fab and 35 anti-Fc fluorescent markers, reveals that both domains are accessible on these aggregates. A two-step mechanism of formation of IgG can be proposed: nucleation and growth of flat, dense islands of IgG on the air-water interface, then transfer into the solution due to forces applied on the solution-air interface

40 during stretching, or breakage of the interfacial film. Of practical importance to design protective agents, we establish a correlation between protection by surfactant additives and the rate of decrease of the surface tension and no specific interfacial interaction between protective surfactants and IgG could be 45 detected. Non specific mechanisms such as, a rapid dynamics of adsorption of surfactants is proposed to bring protection because of the lowering the surface energy stored during transient stretch of interfaces. Rapidly adsorbed surfactants may also efficiently compete with IgG to cover nascent stretched interfaces. These 50 results are not specific to IgG and we expect that they could help to rationalize conditions of handling of the solutions of many proteins.

\section{Acknowledgments}

This work was supported by LFB Biotechnologies ("Laboratoire 55 Français de Fractionnement et des Biotechnologies”) Les Ulis,
France. We thank M. Kouda and B. Bresson (ESPCI), and G. Hallais, Y. Chen (both from Ecole Normale Supérieure) for access to and help with AFM imaging.

\section{Notes and references}

$60{ }^{a}$ Ecole Normale Supérieure, UMR CNRS-ENS-UPMC Paris 068640 Pasteur, 24, rue Lhomond, 75231 Paris Cedex 05, France Corresponding author E-mail : christophe.tribet@ens.fr ${ }^{b}$ LFB, Laboratoire Français de Fractionnement et des Biotechnologies, Les Ulis, France.

$65 †$ Electronic supplementary information (ESI) available: Figure S1: DLS size distribution of IgGp solution as function of the time of rotation with needle; Figure S2: Dropwise flow of solutions inducing antibodies aggregation; Figure S3: AFM evidence of the presence of IgG monolayer at the mica surface; Figure S4: Dynamic surface tension of $1.5 \mathrm{~g} / \mathrm{L}$ 70 antibodies; Figure S5: Fluorescent microscopy observation of IgGp immobilized on mica in the presence of surfactin; Table S1: Characteristic physical parameters of surfactants listed in the order of protective efficiency against IgG aggregation. See DOI: $10.1039 / \mathrm{b} 000000 \mathrm{x} /$

\section{${ }_{75}$ References}

1. V. Sharma, A. Jaishankar, Y. C. Wang and G. H. McKinley, Soft Matter, 2011, 7, 5150-5160.

2. D. E. Dunstan, P. Hamilton-Brown, P. Asimakis, W. Ducker and J. Bertolini, Soft Matter, 2009, 5, 5020-5028.

80 3. M. A. H. Capelle, R. Gurny and T. Arvinte, Eur. J. Pharm. Biopharm., 2007, 65, 131-148.

4. J. S. Philo and T. Arakawa, Curr. Pharm. Biotechnol., 2009, 10, 348351.

5. W. Wang, Int. J. Pharm., 2005, 289, 1-30.

85 6. A. M. Donald, Soft Matter, 2008, 4, 1147-1150.

7. K. Ako, T. Nicolai, D. Durand and G. Brotons, Soft Matter, 2009, 5, 4033-4041.

8. R. Walder and D. K. Schwartz, Soft Matter, 2011, 7, 7616-7622.

9. N. Mahmoudi, M. A. V. Axelos and A. Riaublanc, Soft Matter, 2011, $90 \quad$ 7, 7643-7654.

10. J. Maldonado-Valderrama, A. P. Gunning, P. J. Wilde and V. J. Morris, Soft Matter, 2010, 6, 4908-4915.

11. T. Tran Le, P. Sabatino, B. Heyman, M. Kasinos, H. Hoang Dinh, K. Dewettinck, J. Martins and P. Van der Meeren, Food Hydrocoll., 2011, 25, 594-603.

12. P. Garidel, C. Hoffmann and A. Blume, Biophys. Chem., 2009, 143, 70-78.

13. A. Lahlou, B. Blanchet, M. Carvalho, M. Paul and A. Astier, Ann. Pharm. Fr., 2009, 67, 340-352.

100 14. V. B. Fainerman, A. V. Makievski and R. Miller, Colloids Surf. A, 1994, 87, 61-75.

15. N. A. Mishchuk, S. S. Dukhin, V. B. Fainerman, V. I. Kovalchuk and R. Miller, Colloids Surf. A, 2001, 192, 157-175.

16. N. A. Mishchuk, V. B. Fainerman, V. I. Kovalchuk, R. Miller and S. S. Duhkin, Colloids Surf. A, 2000, 175, 207-216.

17. A. F. H. Ward and L. Tordai, J. Chem. Phys., 1946, 14, 453-461.

18. H.-C. Mahler, R. Müller, W. Friess, A. Delille and S. Matheus, Eur. J. Pharm. Biopharm., 2005, 59, 407-417.

19. A. R. Mackie, A. P. Gunning, P. J. Wilde and V. J. Morris, $110 \quad$ Langmuir, 2000, 16, 8176-8181.

20. J. Maldonado-Valderrama, N. C. Woodward, A. P. Gunning, M. J. Ridout, F. A. Husband, A. R. Mackie, V. J. Morris and P. J. Wilde, Langmuir, 2008, 24, 6759-6767.

21. R. Miller, V. S. Alahverdjieva and V. B. Fainerman, Soft Matter, 115 2008, 4, 1141-1146.

22. R. Miller, V. B. Fainerman, A. V. Makievski, J. Krägel, D. O. Grigoriev, V. N. Kazakov and O. V. Sinyachenko, Adv. Colloid Interface Sci., 2000, 86, 39-82.

23. R. J. Green, T. J. Su, H. Joy and J. R. Lu, Langmuir, 2000, 16, $5797-$ $120 \quad 5805$ 
24. C. Kotsmar, D. O. Grigoriev, F. Xu, E. V. Aksenenko, V. B. Fainerman, M. E. Leser and R. Millert, Langmuir, 2008, 24, 1397713984.

25. A. R. Mackie, A. P. Gunning, P. J. Wilde and V. J. Morris, Langmuir, 2000, 16, 8176-8181.

26. T. D. Gurkov, S. C. Russev, K. D. Danov, I. B. Ivanov and B. Campbell, Langmuir, 2003, 19, 7362-7369.

27. J. Brange, L. Andersen, E. D. Laursen, G. Meyn and E. Rasmussen, J. Pharm. Sci., 1997, 86, 517-525.

10 28. L. C. Gu, E. A. Erdos, H. S. Chiang, T. Calderwood, K. Tsai, G. C. Visor, J. Duffy, W. C. Hsu and L. C. Foster, Pharm. Res., 1991, 8, 485-490.

29. A. M. Tsai, J. H. van Zanten and M. J. Betenbaugh, Biotechnol. Bioeng., 1998, 59, 273-280.

15 30. B. A. Kerwin, M. C. Heller, S. H. Levin and T. W. Randolph, J. Pharm. Sci., 1998, 87, 1062-1068.

31. A. V. Pivovarova, N. A. Chebotareva, I. S. Chernik, N. B. Gusev and D. I. Levitsky, FEBS J., 2007, 274, 5937-5948.

32. J. S. Bee, D. Chiu, S. Sawicki, J. L. Stevenson, K. Chatterjee, E.

20 Freund, J. F. Carpenter and T. W. Randolph, J. Pharm. Sci., 2009, 98, 3218-3238.

33. A. F. Henson, J. R. Mitchell and P. R. Musselwhite, J. Colloid Interface Sci., 1970, 32, 162-165.

34. V. Sluzky, J. A. Tamada, A. M. Klibanov and R. Langer, Proc. Natl. Acad. Sci. U.S.A., 1991, 88, 9377-9381.

35. W. Wang, Y. J. Wang and D. Q. Wang, Int. J. Pharm., 2008, 347, 3138.

36. J. Maldonado-Valderrama and D. Langevin, J. Phys. Chem. B, 2008, 112, 3989-3996

30 37. J. G. Hansted, P. L. Wejse, H. Bertelsen and D. E. Otzen, Biochim. Biophys. Acta, 2011, 1814, 713-723.

38. R. Kun, L. Kis and I. Dékány, Colloids Surf. B, 2010, 79, 61-68. 